\title{
Optimal Control of District Heating Systems using Dynamic Simulation and Mixed Integer Linear Programming
}

\author{
Loïc Giraud Massinissa Merabet Roland Baviere Mathieu Vallée \\ Univ. Grenoble Alpes, INES, F-73375 Le Bourget du Lac, France \\ CEA, LITEN, 17, Rue des Martyrs, F-38054 Grenoble, France, roland.baviere@ cea.fr
}

\begin{abstract}
This paper presents the development of a new advanced control method suitable for variable temperature District Heating Systems (DHS). The proposed controller determines optimal planning for the on/off status and power of the heat generators as well as for the supply temperature and differential pressure at the production plant level. Compared to existing methods, the original features of the developed solution are to fully exploit the thermal storage capacity of the network and to determine the best compromise between pumping costs and heat losses. A numerical case study based on a representative DHS is used to evaluate the method over a heating season (5 months). Results show that our method reduces production costs up to $8.3 \%$ when compared to a more classical controller. Moreover, the observed computing time is compatible with the requirements of the receding time horizon principle, ensuring that the method is tractable on real DHS.
\end{abstract}

Keywords: $\quad$ District Heating, Model Predictive Control, Dynamic Simulation, Mixed Integer Linear Programming

\section{Introduction}

Nowadays, many research works devoted to District Heating Systems (DHS) are performed on low and very low temperature systems, mainly because of their energy performance and their ability to use renewable energy sources. However, High Temperature District Heating Systems (HTDHS) represent an important share of the existent systems in Europe. For instance, systems with temperature over $110{ }^{\circ} \mathrm{C}$ account for more than 50 $\%$ of the heat delivered by French DHS (SNCU, 2013).

The energy load of HTDHS is generally supplied by numerous generators and fuels. On the other hand, their distribution network usually bears large variations in temperature and differential pressure. Thus, HTDHS are affected by non-constant production costs yet they natively comprise important thermal storage capacities, i.e. network storage, if an adequate supply temperature control is used. Additionally, HTDHS are subject to complex dynamic behaviors originating both from the variability of the demand and the significant temperature transportation delay. Finally, heat can be delivered to the consumers using various combinations of temperature and mass flow rate. Lowering network temperature would limit the thermal losses; however, the mass flow rate shall increase in order to maintain the same heat flow, and this will cause pumping work to rise. Contrary to what is generally considered, in many practical situations, particularly recurrent in HTDHS, the optimal balance between pumping work and heat losses may be obtained with high supply temperature and low differential pressure.

As pointed in (Lund, 2014), the intelligent control for optimal operation is a future challenge for the improvement of DHS. Due to its complexity and high parameters combinatorics, the determination of optimal production and distribution plans in DHS is difficult, if not impossible, when solely based on empirical laws and/or expert judgement. In this context, decision support/making tools relying on a Model Predictive Control (MPC) scheme are very useful. Despite significant progress, there is still an important room for improvement in this domain.

This paper focuses on the optimal control of pressurized water DHS. For this application, we have developed and tested an algorithm that optimizes, given a load prediction, the use of production means as well as supply temperature and differential pressure. Compared to existing methods, the original features of the developed solution are, firstly, to fully exploit the thermal storage capacity of the network. Secondly, our controller is suitable for determining the best possible combination between electrical costs for pumping and heat production costs compensating distribution losses. Though generic, the proposed control method is particularly adapted to existing HTDHS.

Our controller is based on a MPC scheme. At each time step, a dynamic non-linear model of the distribution network is simulated over a finite timehorizon. In the present work, this model is built using the equation-based object-oriented language Modelica along with the simulation platform Dymola and an inhouse component model library named DistrictHeating (Giraud b), 2015). The simulation results are then used to formulate a linearized model of the distribution network. Combined with other linear constraints representing the technical limitations of the different 
pieces of equipment and with a linear cost function, the model forms a Mixed Integer Linear Program (MILP). In a last step, this program is solved yielding the optimal trajectories for the various control variables of the problem.

In section 2, we present a literature review on advanced control for DHS. Section 3 firstly describes the algorithmic aspects of the proposed controller, secondly presents the non-linear network model used for dynamic simulation and finally details the formulation of the linear optimization problem. A case study consisting of a virtual yet representative HTDHS is then described in section 4. In section 5, the simulation results obtained for various controllers over a heating season are presented and discussed. Section 6 includes the conclusions and perspectives of our study.

\section{Existing DHS Control Methods}

The approach currently used to determine the control variables of DHS (e.g. supply temperature, differential pressure, load distribution between different heat production units ...) often relies on heuristic methods, i.e. a formalization of common sense, simple logic or expert judgement. As an example, we can recall here the determination of supply temperature using a single or even multi-variable heating curve. Though simple to implement and robust with respect to production or demand uncertainties, the efficiency of such control methods is always limited when applied to a system comprising several sources, variable energy purchase prices and energy storage capacity. This is partly due to the fact that anticipative control strategies are difficult if not impossible to formulate in this framework. Another difficulty is that production goals may be multiple and conflicting (e.g. power and heat generation in combined heat-and-power units).

To overcome the aforementioned difficulties, an MPC scheme is an interesting alternative. The MPC approach consists of a load prediction module and an optimization procedure used to determine the best possible path for control variables, i.e. the one minimizing an objective function while meeting different technical and operational constraints. Depending on the formulation of the quantitative objective to minimize, operating costs and/or $\mathrm{CO}_{2}$ content of the delivered heat may be significantly improved.

However, building an MPC scheme to control a DHS is a complex task. This explains why most previous works done on this subject only consider some parts of the problem. On the one hand, numerous studies deal with production optimization only, i.e. they address the unit commitment and load dispatch problems. In (Eriksonn, 1994), the author determines the heat power planning for each production unit considering starting costs, minimal load of each generator and bounds for heat power ramp rates. This approach is mostly used in studies interested in combined heat and power plants since electricity must be produced when it is the most profitable. On the other hand, several works only consider the supply temperature determination. Important features here are to reduce heat losses and to use the network capacity for heat storage. For instance, the supply temperature is optimized in (Nielsen, 2005) using the Finite Impulse Response method to linearize a dynamic distribution network model and then to solve the linear optimization problem. Few works study both the load dispatch problem and the supply temperature determination. The integer variables, representing for instance the heat generators' statuses, are then not taken into account in these cases in order to reduce the combinatory.



Figure 1: Proposed optimal control algorithm for DHS.

More recently, both the supply temperature and heat power planning have been determined in order to minimize the production costs yet without considering the time delays in the network (Fang, 2015). Another possible approach, quite popular in the Modelica community, consists in modeling, formulating and solving a dynamic optimization problem using the JModelica.org tools (Akesson, 2011). Following this method, (Runvik, 2015) also solve a short-term production planning problem for a DHS using a twosteps optimization procedure including production and distribution variables. However, due to prohibitive computational costs, the network representation only includes three customers. 
In conclusion of this review, no method has been identified in the literature that adequately optimizes the production and the distribution considering network storage for DHS. In the following, we present such a method and study its benefits on a representative HTDH.

\section{Optimal Control of DHS}

In this section, we describe the optimal control method we designed. The objective is to determine the optimal trajectory for the control variables over a defined anticipation horizon. Taking into account the relatively slow dynamics of a DHS requires anticipation, i.e. using load predictions and optimizing of control variables so that the system produces the desired effects in the future. Additionally, periodically revising the optimization is mandatory in order to cope with load prediction uncertainties.

To address these challenges, we combine a dynamic nonlinear model of the DHS with linear optimization methods, similarly to other works like those of (Benonysson, 1991) and (Sandou, 2006). For each anticipation horizon, our system encompasses both production and distribution optimization and controls heat powers, generators' statuses, supply temperature and differential pressure. To guarantee the applicability of the control trajectories, the optimization is constrained by the technical limits of the DHS's pieces of equipment.

This section first gives a general description of the algorithm, then details the dynamic nonlinear model, which we illustrate more specifically in the case study. It ends with a description of the linear optimization problem's formulation.

\subsection{General Description}

Figure 1 depicts the proposed algorithm. The dynamic nonlinear network model is first simulated using initial guesses for the distribution control variables, namely the supply temperature and differential pressure at production plants. We then extract relevant input data for the optimization problem and we formulate a linear relaxation of the optimization problem using the MILP formalism. The optimizer finally computes a new set of control variables. Iterations between the dynamic network model and the MILP optimizer are conducted until convergence is reached, using a criteria defined by a threshold on the supply temperature increment.

We then periodically revise the optimization using the receding time horizon. At time $t$, the optimization procedure is performed for the predictive horizon $t+N_{t}$ yet only the first output values for time slot $[t: t+P r]$ are applied to the system. At time $t+P r$, the calculation is repeated for the optimization horizon $t+P r+N_{t}$. This algorithm is illustrated in Figure 2 in a situation where the receding horizon $P r$ is chosen equal to 1 .

\subsection{The dynamic distribution network model}

The proposed algorithm requires the simulation of a dynamic model representing the distribution network.

Figure 3 depicts the mesh-free layout of the distribution network considered in the case study. Since we study our control strategy on a mid-scale DHS, our dynamic network model is based on a detailed physical representation of the system by gathering component models that we previously developed and validated. The components models are taken from an in-house Modelica library named DistrictHeating and presented in (Giraud b), 2015). The heat generators are represented considering equivalent heat and momentum sources. Moreover, the model enables the control of supply temperature and differential pressure at the production plant level. The distribution pipe model that we use is based on the method of characteristics, also called node method in the specific DHS related literature see (Benonysson, 1991). This model accounts for heat propagation delays, heat losses, tube thermal inertia and pressure losses. Concerning the substation representation, we use an explicit model comprising a
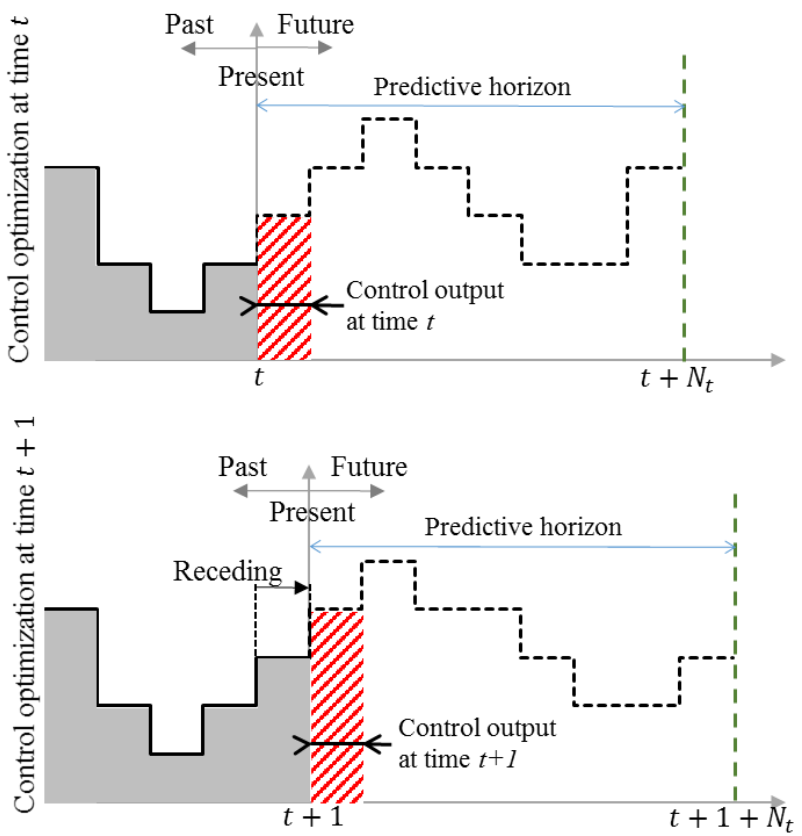

Figure 2: Receding horizon principle

heat exchanger, a regulation valve and an ideal controller (see (Giraud a), 2015) for details).

\subsection{Formulation of the Linear Optimization Problem}

The linear optimization problem is an approximation, or relaxation, of the complete, strongly nonlinear problem that would take into account all the physical and technical constraints of the system. However, 
formulating the linear problem using appropriate assumptions yields usable results in a limited computating time. This section details the formulation of the linear problem we adopted, which includes the MILP variables, the cost function to minimize, the technical limits of the DHS, the critical curve describing consumer's constraints and a linearized model of the distribution network.

\subsubsection{MILP variables}

The optimization variables of the problem are identified by an asterisk and typed in bold to better readability. All of them are time discretized and considered constant over one time step.

\section{Continuous variables}

$\dot{\boldsymbol{Q}}_{\boldsymbol{g}}^{*}(t)$ : Represents the heat power produced by each generator $g$ at the time $t$.

$\boldsymbol{T}_{\text {sup }}^{*}(t)$ : Represents the supply temperature in the network at the time $t$.

$\boldsymbol{m}_{\text {tot }}^{*}(t)$ : Represents the total mass flow rate at the production plant at the time $t$.

$\Delta \boldsymbol{P}_{\boldsymbol{p m p}}^{*}(\mathrm{t}):$ Represents the differential pressure at the production plant at the time $t$.

$\boldsymbol{W}_{p m p}^{*}(\mathrm{t}):$ Represents the pump work at the time $t$.

Binary variables

$\boldsymbol{Y}_{\boldsymbol{g}}^{*}(t)$ : Represents the on/off status of each generator, i.e. it is equal to one when generator $g$ is $o n$ and to zero otherwise.

$\boldsymbol{X}_{\boldsymbol{g}}^{*}(t)$ : Identifies the timing of each generator startup, i.e. it is equal to one only when $\dot{\boldsymbol{Y}}_{\boldsymbol{g}}^{*}$ switches from 0 to 1 and to zero otherwise.

\subsubsection{The cost function}

The function to be minimized, presented in expression (1), reflects the integral of operational costs over a finite time-horizon. The first term in equation (1) represents the fuel consumption and it is thus proportional to the
$C_{g}^{t h}$ parameters standing for fuel prices. The second term accounts for specific costs linked to generator start-up and they are therefore proportional to fixed monetary amounts denoted $C_{g}^{o f f / o n}$. The last term accounts for electricity consumption due to pump's operation. It is therefore proportional to a time variable electricity purchase price hereafter denoted $C^{e l}(t)$. Pumping and heat generation efficiencies are accounted for respectively using the $\eta_{p m p}$ and $\eta_{g}$ constant parameters.

$$
\begin{gathered}
\sum_{t . t+P r}\left(\sum _ { g } \left(\frac{C_{g}^{t h}}{\eta_{g}} \cdot \dot{\boldsymbol{Q}}_{g}^{*}(t) \cdot d t+C_{g}^{o f f / o n}\right.\right. \\
\left.\cdot \boldsymbol{X}_{g}^{*}(t)\right)+\frac{C^{e l}(t)}{\eta_{p m p}} \cdot \dot{\boldsymbol{W}}_{p m p}^{*}(t) \\
\cdot d t)
\end{gathered}
$$

To guarantee the applicability of its outcomes, the minimization calculation must be performed in the presence of linear constraints on the optimization variables. Inequality constraints will be presented first. In a second step we present the equality constraints accounting for the mass, energy and momentum balance equations governing the relations between the operating variables.

\subsubsection{Inequality constraints}

Several continuous variable are considered with lower and upper bounds representing physical limitations of DHS components as presented in inequalities (2), (3) and (4). Each parameter noted with a $\min$ or $\max$ superscript is a known and fixed parameter of the problem.

$$
\begin{gathered}
T_{\text {sup }}^{\min } \leq \boldsymbol{T}_{\text {sup }}^{*}(t) \leq T_{\text {sup }}^{\max } \\
\Delta P_{p m p}^{\min } \leq \boldsymbol{\Delta} \boldsymbol{P}_{\text {pmp }}^{*}(t) \leq \Delta P_{p m p}^{\max }
\end{gathered}
$$

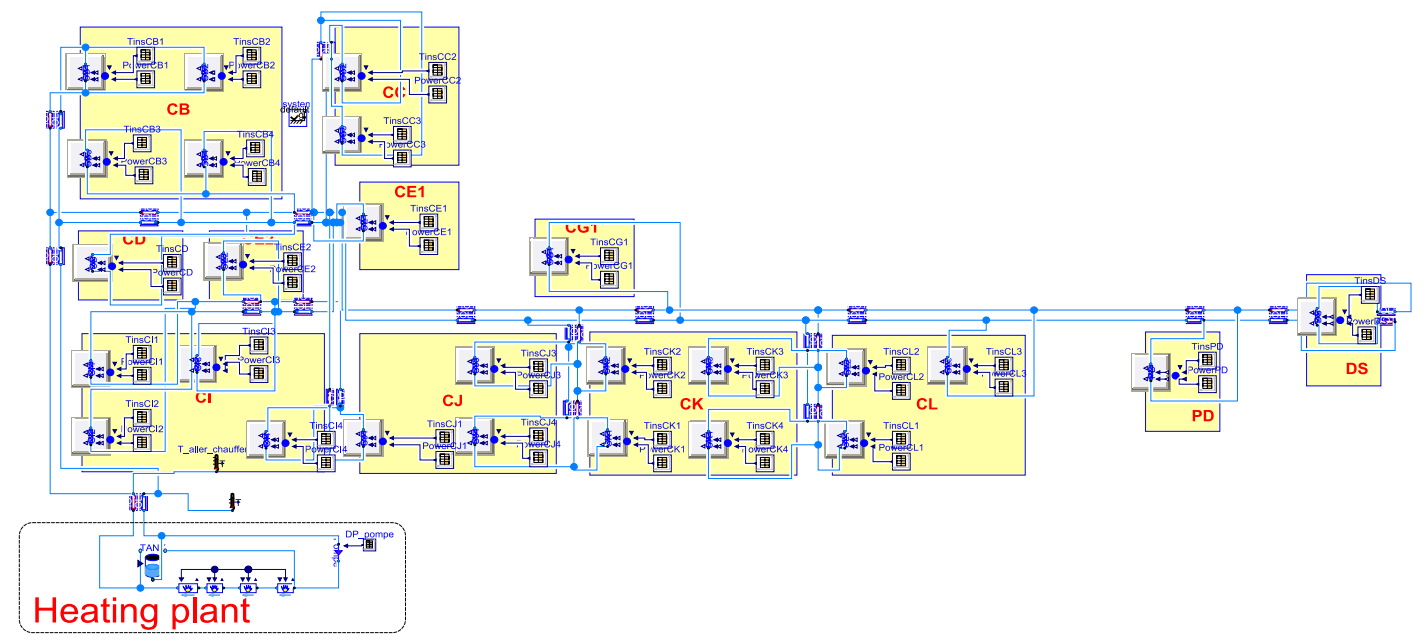

Figure 3: Layout of the distribution network case study in Modelica/Dymola. 


$$
\dot{m}_{\text {tot }}^{\min } \leq \dot{\boldsymbol{m}}_{\text {tot }}^{*}(t) \leq \dot{m}_{\text {tot }}^{\max }
$$

To limit thermal fatigue, we bound supply temperature and heat power variations with expressions



expression (8) relating $\dot{Q}_{c}$, the primary mass flow rate $\dot{m}_{c}$, the primary inlet and outlet temperatures $\left(T_{c, \text { in }}\right.$ and $\left.T_{c, \text { out }}\right)$ and the fluid specific heat capacity $C p$.

Figure 4: Consumer critical temperature as a function of the differential pressure and heat demand (left) Cut-plane for a fixed heat demand (right).

of the following type, written for a sample variable $\boldsymbol{Z}^{*}$ :

$$
-\Delta Z^{\max } \leq \boldsymbol{Z}^{*}(t)-\boldsymbol{Z}^{*}(t-1) \leq \Delta Z^{\max }
$$

Inequalities (6) are used to confine $\dot{\boldsymbol{Q}}_{\boldsymbol{g}}^{*}$ between $\dot{Q}_{g}^{\min }$ and $\dot{Q}_{g}^{\max }$ when generator $g$ is started. Otherwise, $\dot{\boldsymbol{Q}}_{\boldsymbol{g}}^{*}$ is set to 0 .

$$
\boldsymbol{Y}_{\boldsymbol{g}}^{*}(t) \cdot \dot{Q}_{g}^{\min } \leq \dot{\boldsymbol{Q}}_{\boldsymbol{g}}^{*}(t) \leq \boldsymbol{Y}_{\boldsymbol{g}}^{*}(t) \cdot \dot{Q}_{g}^{\max }
$$

Finally, inequalities (7) are considered to define the timing of each generator start-up, i.e. variable $\dot{\boldsymbol{X}}_{\boldsymbol{g}}^{*}$.

$$
\boldsymbol{Y}_{\boldsymbol{g}}^{*}(t)-\boldsymbol{Y}_{\boldsymbol{g}}^{*}(t-1) \leq \dot{\boldsymbol{X}}_{\boldsymbol{g}}^{*}(t) \leq \boldsymbol{Y}_{\boldsymbol{g}}^{*}(t)
$$

\subsubsection{Critical conditions to supply heat demand}

Supplying the requested heat demand to a DHS customer is only possible when the local network temperature exceeds a threshold called the critical temperature and hereafter denoted $T_{c, i n}^{c r i t}(t)$. The present section firstly discusses how to derive the formula used to evaluate $T_{c, i n}^{c r i t}(t)$ and secondly presents the linear inequality constraints necessary in the MILP problem to guarantee that heat demand is fulfilled.

We consider in this study that the substations are of the indirectly connected type and that they are composed of one counter-flow heat-exchanger, free of any by-pass, and a primary control valve used to regulate the building heating system temperature at a requested level. For such system, as long as the consumer heat demand $\dot{Q}_{c}$ is fulfilled, a static energy balance applied on the primary side of the heat exchanger yields the mathematical

$$
\dot{m}_{c}(t)=\frac{\dot{Q}_{c}(t)}{C p \cdot\left(T_{c, \text { in }}(t)-T_{c, \text { out }}(t)\right)}
$$

On the other hand, $\dot{m}_{c}$ cannot exceed the value reached when the primary control valve is fully open. Such maximal value, denoted $\dot{m}_{c}^{\max }(t)$, can be calculated assuming a quadratic dependency between the local differential pressure of the network, namely $\Delta P_{c}$, and the mass flow rate. This is shown in equation (9) used for valve modeling. In this equation, $\dot{m}_{c}^{\text {nom }}$ and $\Delta P_{c}^{\text {nom }}$ are nominal values of the primary mass flow rate and the differential pressure.

$$
\dot{m}_{c}^{\max }(t)=\dot{m}_{c}^{\text {nom }} \cdot \sqrt{\frac{\Delta P_{c}(t)}{\Delta P_{c}^{\text {nom }}}}
$$

The critical temperature is obtained by assuming that the demand is fulfilled while $\dot{m}_{c}$ equals $\dot{m}_{c}^{\max }$. Thus, combining equations (8) and (9) leads to expression (10) for the critical temperature:

$$
T_{c, \text { in }}^{\text {crit }}(t)=T_{c, \text { out }}(t)+\frac{\dot{Q}_{c}(t)}{C p \cdot \dot{m}_{c}^{\text {nom }} \cdot \sqrt{\frac{\Delta P_{c}(t)}{\Delta P_{c}^{\text {nom }}}}}
$$

At this stage, it is worth mentioning that an additional heat-exchanger model is requested to evaluate formula (10) since the primary outlet temperature, namely $T_{c, \text { out }}(t)$, has not been determined yet. In our study, the Logarithmic Mean Temperature Difference method is used for this purpose. 
As a consequence, heat demand of consumer $c$ will only be satisfied if local network conditions in terms of temperature and differential pressure are above critical values shown in Figure 4, i.e. if inequality (11) is verified:

$$
T_{c, \text { in }}(t) \geq T_{c, \text { out }}(t)+\frac{\dot{Q}_{c}(t)}{C p \cdot \dot{m}_{c}^{\text {nom }} \cdot \sqrt{\frac{\Delta P_{c}(t)}{\Delta P_{c}^{\text {nom }}}}}
$$

This last expression is adapted to our problem using piecewise linear approximations compatible with the MILP formalism:

$$
T_{c, i n}(t) \geq \operatorname{fin}_{c, l}\left(\Delta P_{c}(t)\right) \quad l=1, \ldots, L
$$

In expression (12), the $\operatorname{flin}_{c, l}$ functions are a set of $L$ linear functions approximating the critical temperature calculated from the right hand-side of expression (11).

Bearing in mind that the algorithm aims at producing optimal planning for the supply temperature and the differential pressure, we then consider linear relations between the variables at the consumers' sides and those at the production plant level where the control variables are applied. The heat propagation equation (13), detailed in (Benonysson, 1991), is used to express the consumers inlet temperature $T_{c, i n}$ as a function of the supply temperature $\boldsymbol{T}_{\text {sup }}^{*}$. It considers a propagation time delay $\tau_{c}$ and heat losses using the $\tau_{\text {therm,c }}$ thermal time constant and the $T_{\text {ext }}$ parameter representing the ambient temperature surrounding the distribution pipes.

$$
\begin{aligned}
T_{c, \text { in }}(t)=T_{\text {ext }}+ & \left(\boldsymbol{T}_{\text {sup }}^{*}\left(t-\tau_{c}(t)\right)-T_{\text {ext }}\right) \\
& \cdot e^{-\frac{\tau_{c}(t)}{\tau_{\text {therm }, c}}}
\end{aligned}
$$

At this point, one can note that temperature propagation delay $\tau_{c}$ introduces nonlinearities into the optimization problem. Thus, propagation delays $\tau_{c}$ are not handled directly in the MILP problem but are provided by the dynamic model presented in section 3.2.

For the differential pressure losses, a linearized relation, well verified on the Grenoble DHS, is considered as shown in expression (14):

$$
\Delta P_{c}(t)=\Delta \boldsymbol{P}_{p m p}^{*}(t)-K_{c} \cdot \boldsymbol{m}_{\text {tot }}^{*}(t)
$$

Expression (12) is then re-written using (13) and (14) to introduce the $\boldsymbol{T}_{\text {sup }}^{*}, \boldsymbol{m}_{\text {tot }}^{*}$ and $\boldsymbol{\Delta} \boldsymbol{P}_{\text {pmp }}^{*}$ optimization variables. This yields the linear inequality constraints (15) that are used in our MILP problem to guarantee that consumer satisfaction is not sacrificed.


Figure 5: Evolutions over the heating season of the normalized heat demand (top) and external temperature (bottom). 


$$
\begin{array}{r}
T_{\text {ext }}+\left(\boldsymbol{T}_{\text {sup }}^{*}(t-\right. \\
\left.\left.\tau_{c}(t)\right)-T_{\text {ext }}\right) \cdot e^{-\frac{\tau_{c}(t)}{\tau_{\text {therm }, c}}} \\
\geq \operatorname{flin}_{c, l}\left(\Delta \boldsymbol{P}_{\text {pmp }}^{*}(t)-K_{c}\right. \\
\left.\cdot \boldsymbol{m}_{\text {tot }}^{*}(t)\right) \quad l=1, \ldots, L
\end{array}
$$

\subsubsection{The linearized distribution network model}

The relations between the optimization variables accounting for distribution network mass, momentum and energy conservation laws are considered thanks to a set of linearized equality constraints that are presented in this section.

First, the pumping work $\dot{\boldsymbol{W}}_{\boldsymbol{p} \boldsymbol{m} \boldsymbol{p}}^{*}$ appearing in the cost function (1) is expressed linearly using a first order Taylor expansion limited to differential pressure and mass flow rate variations. The fluid density $\rho$ are therefore assumed constant, which leads to expression (16):

$$
\begin{aligned}
& \dot{\boldsymbol{W}}_{\boldsymbol{p} \boldsymbol{m} \boldsymbol{p}}^{*}(t) \\
& =\frac{\dot{m}_{t o t}(t) \cdot \boldsymbol{\Delta} \boldsymbol{P}_{\boldsymbol{p m p}}^{*}(t)+\dot{\boldsymbol{m}}_{\text {tot }}^{*}(t) \cdot \Delta P_{p m p}(t)}{\rho} \\
& -\frac{\dot{m}_{t o t}(t) \cdot \Delta P_{p m p}(t)}{\rho}
\end{aligned}
$$

In this last expression, $\Delta P_{p m p}(t), \dot{m}_{t o t}(t)$ and $\eta_{p m p}$ are provided by the dynamic simulation model described in section 3.2.

Second, a linearized version of the network energy balance is obtained. This point is crucial if one wants to benefit from the possibility to store heat directly in the distribution network. By following the derivation presented in (Giraud, 2016), the subsequent expression can be obtained:

$$
\begin{gathered}
\sum_{g} \dot{\boldsymbol{Q}}_{\boldsymbol{g}}^{*}(t)=\sum_{c} \dot{Q}_{c}(t) \cdot\left[1+\boldsymbol{F}_{\boldsymbol{T}}^{*}(t)\right] \\
-\dot{\boldsymbol{W}}_{\boldsymbol{p} \boldsymbol{m} \boldsymbol{p}}^{*}(t)
\end{gathered}
$$

In expression (17), $\boldsymbol{F}_{T}^{*}(t)$ is a modulation term that depends on past and present values of supply temperature.

\subsubsection{Summary}

Our MILP DHS production and distribution optimizer is composed of a linear cost function (see equation (1)) subject to linear equality (see equations (16) and (17)) and inequality (see equations (2)-(7), (15)) constraints representing the physical conservation laws and the technical limitations of the DHS.

\section{The case study}

The operation of our advanced controller has been evaluated by simulation means relying on a representative case-study. This section explains how the case-study has been designed and it details the models composing it.

The CCIAG company and our research group are currently involved in a joint research program devoted to the development of advanced decision support/making tools for operational management of DHS. CCIAG operates the second largest DHS in France in the city of Grenoble. This system yearly delivers $900 \mathrm{GWh}$ of heat using $225 \mathrm{~km}$ of distribution pipes and liquid pressurized water as heat carrier fluid. This system is actually managed using variable supply temperatures and differential pressures respectively ranging from $110{ }^{\circ} \mathrm{C}$ to $180{ }^{\circ} \mathrm{C}$ and 5 to 15 bars. These features are similar to systems in other French and European cities (e.g. Metz, Chambéry, Vienna, Warsaw...). Therefore, we have built the case-study used in the present paper upon the Grenoble HTDH system. However, in order to limit the modelling work during the first stages of our research project we considered only a portion of the Grenoble distribution network.

On the production side, we have considered 15 heat production units as it is representative of the installed capacity in Grenoble. All the 15 heat production units form a unique production plant represented in the dynamic model by equivalent heat and momentum sources. The sample network serves 26 heat consumers modelled using load profiles taken from an historical database (15 min sampling period) provided by CCIAG. The substation models are parametrized using the dimensioning rules applied on the Grenoble DHS.

To increase the relevance of the model, the main parameters used to model the production units in the MILP model have been proposed by CCIAG, our industrial partner in the project. For confidentiality purposes, the fuel prices denoted $C_{g}^{t h}$, the cost of a generator start up denoted $C_{g}^{o f f / o n}$ and the daily electricity price profile used for pump operation, denoted $C^{e l}$ are not reported here.

\subsection{Simulation settings}

The simulation period covers the full 2013/2014 French heating season, i.e. from November 2013 to mid-April 2014. The heat demand normalized by the installed heat power capacity (designed for an external temperature of $-11^{\circ} \mathrm{C}$ ) and the external temperature over that period are shown in Figure 5. Outside this period, low cost heat from the waste incineration unit is produced in excess of demand thereby limiting the usefulness of advanced control strategy. Moreover, the heating season represents over $80 \%$ of annual production costs.

The simulations were conducted with an elementary time step of 15 minutes. The optimization time-horizon is set to 24 hours and the receding horizon is fixed at 6 hours. For control in real conditions, affected by many sources of uncertainty (e.g. load prediction errors ...), 
the receding horizon would be chosen equal to the 15 min elementary time step to increase robustness and stability. A complementary study on this topic is currently under investigation.

A $1 \mathrm{~K}$ threshold on the supply temperature increment is used to decide that convergence between the dynamic model and the MILP problem is reached. We set the relative MIP gap tolerance to 0.03 . That instructs CPLEX to stop as soon as it has found a feasible integer solution proved to be within three percent of optimal. This tolerance is less than the error due to the uncertainties of the predictions. There is no relaxation in our resolution method since no approximation scheme is used, so we always find a solution within $3 \%$ from the optimal which is a global minima. It could be interesting to compute a relaxation of the MIP and compare the exact and the approximate resolutions, but the good performance of our model in terms of computational time makes the exact resolution compatible with the online application of our controller on a real DHS.

\subsection{Implementation}

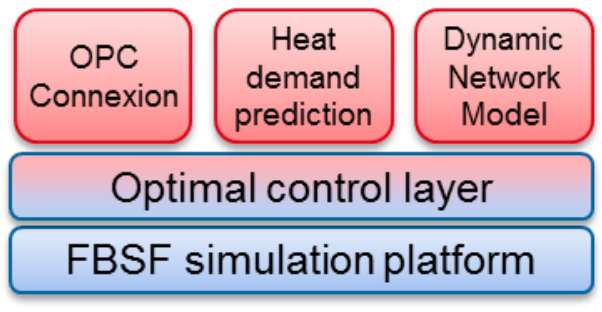

Figure 7: Architecture of the PEGASE optimal control framework

The overall algorithm is programmed using an in-house optimal control framework named PEGASE. PEGASE is based on the FBSF platform developed by the L3S company (L3S, 2016). FBSF enables multi-models simulation based on the FMI 2.0 co-simulation standard.

Figure 6 illustrates the architecture of the PEGASE optimal control framework. The lower layer is the FBSF simulation platform, which provides the basic services for running multi-model simulations. The middle layer is the optimal control layer, which performs the optimal control algorithm presented in the previous sections. The upper layer contains the dynamic simulation models used by the optimal control layer, as well as other prediction models and generic OPC connectivity services.

For the application described in this paper, we use only one dynamic simulation model, which consists of the Modelica model of the distribution network. This model is converted into a 2.0 co-simulation FMU by DYMOLA 2017. For other applications, several dynamic simulation models can be used together, either in the form of FMU or with FBSF-specific $\mathrm{C}++$ code.

Within the optimal control layer, we express the MILP optimization problem using an in-house $\mathrm{C}++$ code and solve it relying on CPLEX (IBM, 2009).
CPLEX can easily and quickly solve numerous problems with high combinatory owing to parallelization and application of the branch and bound method to reduce the search space (Brah, 1991). As a result, the computational time is generally lower than with other MILP solvers.

\section{Results and Discussion}

The simulation results obtained over the heating season are presented and discussed in this section. The numerical performance of the controller are also described. The current limitations of the proposed controller are finally presented at the end of this section.

For evaluation purposes, we compared the performance of our advanced controller to a more classical controller based on expert laws. This controller is still a popular method used in many existing systems owing to its simplicity and robustness. The standard controller is based on the piling method for the production planning. On the distribution side, the supply temperature is determined using a static heating curve while the differential pressure is maximized. To limit a chattering effect on the on/off status of heat production units, a hysteresis time-dependence is considered in the determination of generators starts and stops.



Figure 6: Normalized production costs over the 2013/2014 heating season for a standard (1) and an optimal controller (2).

As displayed in Figure 7, results point that our method significantly reduces production costs both with and without the consideration of uncertainties. The production cost's decrease is explained in the following section.

Table 1. Computational time and iterations for a 24 hours predictive horizon.

\begin{tabular}{|c|c|c|c|}
\hline \multicolumn{2}{|c|}{ Computational time } & \multicolumn{2}{c|}{ Number of iterations } \\
\hline Mean & Max & Mean & Max \\
\hline $37.8 \mathrm{~s}$ & $273.7 \mathrm{~s}$ & 3 & 29 \\
\hline
\end{tabular}

On the production side, the optimal controller often keeps several peak generators at minimal load to anticipate future peak demands and avoid start-up costs when the produced heat is low (particularly during nights). As a consequence, the number of generator 




Figure 8: Snapshot of a dynamic simulation of the Grenoble DHS within the PEGASE

startups over the season is significantly reduced between the expert law control and the optimal controller.

On the distribution side, due to the optimization of $T_{\text {sup }}$ and $\Delta P_{p m p}$, results are very specific for the optimal controller. On the one hand, the supply temperature is often minimized and the differential pressure is maximized. As a result, the heat losses are reduced and the pumping work is increased for a global energy consumption reduction. This is due to the low pumping costs compared to the heat production costs encountered in this case study. On the other hand, our optimal controller is able to use the storage capacity of the network to anticipate future peak demands thereby increasing the use of base generators and avoiding the use of additional and expensive heat generators. To benefit from the network storage capacity, our control strategy increases the supply temperature prior to a peak demand. Accordingly, differential pressure is decreased without impacting the supplied heat demand. As a consequence of using the network storage capacity, the number of generators' startups is further decreased.

Table 1 presents the mean and maximal values of computational costs and iterations for a 24 hour predictive horizon. Using a $15 \mathrm{~min}$ time step, the optimization problem contains 5430 variables including 2460 binary variables and 5530 constraints. The mean and maximal computational time are respectively less than $40 \mathrm{~s}$ and about 4 minutes. These figures are compatible with the on-line application of our controller on a real DHS.

The controller, as described in the present paper, is currently restricted to DHS comprising one single heating plant feeding a non-meshed network. Application of the method to a multiple supply points DHS and a meshed network is the subject of ongoing work. Another point worth mentioning is that the number of constraints of the MILP problem grows linearly with respect to the number of consumers (see inequality (15)). Thus, the application of the proposed method to large-scale DHS impose to consider a set of critical consumers in the network. As suggested in (Nielsen, 2005), such consumers may be selected so that if the $T-\Delta P$ (see inequality (15)) requirements for them are satisfied then the requirements for all consumers are satisfied. It has been verified that the current CPLEX MILP solver could handle problems comprising a set of several hundreds of critical consumers. This testing can be considered positive with respect to the scalability of the proposed controller.

\section{Conclusions}

In this paper, we present a new control method for DHS management which simultaneously optimizes the production and the distribution variables. For each anticipation horizon, an optimized planning for the status and power of each generator as well as for the 
supply temperature and the differential pressure is proposed. Based on the heat demand prediction of each consumer or group of consumers, our controller determines autonomously the combination of supply temperature and differential pressure necessary to supply the heat demand. The method is based on an algorithm minimizing the production costs and respecting a family of constraints representing the conservation laws and the physical limitations of the generators and the distribution network. We consider the nonlinearities of the distribution network thanks to an iterative method between the dynamic network simulation and the optimizer. Once implemented on a DHS, this generic control strategy will autonomously select the best compromise among the control variables to minimize the production costs.

We also compared the proposed method to a more classical controller based on expert law. The comparison is based on the simulation over a heating season of a virtual DHS representative of the Grenoble case. Results show that our global optimization method improves the seasonal production costs by more than 8 $\%$ compared to empirical methods. The proposed controller decreases the production costs by taking advantage of the network storage capacity. The use of expensive peak heat generators is then minimized whereas base heat generators operation is maximized.

The distribution network dynamic model used in the present study was built by gathering components taken from the Modelica DistrictHeating modelling library. However, due to efficiency issues well described in (Casella, 2015), such modelling approach is currently not suitable for the representation of large-scale DHS comprising thousands of consumers and encompassing several hundreds of kilometers of distribution pipes. For such systems, we developed a dedicated $\mathrm{C}++$ simulation code, not detailed in the present paper, and applied it to the Grenoble DHS (see Figure 8). This last development paved the way to the application and testing of our optimal controller on the $400 \mathrm{MW}_{\text {th }}$ Grenoble DHS during the 2016-2017 heating season.

\section{Acknowledgements}

The authors sincerely wish to thank Elise Le Goff, Nicolas Giraud and Philippe Clolot from CCIAG, our industrial partner in the project, for the many stimulating exchanges and for providing real-life data from the Grenoble network. We would also like to acknowledge the financial support of CCIAG for the joint research program and of ADEME for the PhD of Loïc Giraud.

\section{References}

Akesson, J., Faber R., Laird C.D., Prolb K. Tummescheit H., Velut S., Zhu Y., "Models of a post-combustion absorption unit for simulation, optimization and non-linear model predictive control schemes", Proc. $8^{\text {th }}$ Modelica Conference, Dresden, Germany, March 20-22, 2011
Brah S. A. and Hunsucker J. L., "Branch and bound algorithm for the flow shop with multiple processors," Eur. J. Oper. Res., no. 51, pp. 88-89, 1991.

Casella F., "Simulation of Large-Scale Models in Modelica: State of the Art and Future Perspectives", Proc. 11 ${ }^{\text {th }}$ International Modelica Conf., Sept 21-23 2015, Versailles, France

Donald C. Augustin, Mark S. Fineberg, Bruce B. Johnson, Robert N. Linebarger, F. John Sansom, and Jon C. Strauss. The SCi Continuous System Simulation Language (CSSL). Simulation, No 9, pp. 281-303, 1967.

Benonysson A., Bøhm B., and Ravn H.F., "Operational optimization in a district heating," Energy Convers. Manag., vol. 36, no. 5, pp. 297-314, 1995

Eriksson H., "Short Term Operation of District Heating Systems: An Application of Mathematical Programming" Doctoral thesis, Chalmers University of Technology, 1994.

Fang T. and Lahdelma R., "Genetic optimization of multiplant heat production in district heating networks," Appl. Energy, vol. 159, pp. 610-619, Dec. 2015.

Giraud L., Bavière R., Paulus C., Vallée M., and Robin J.-F., "Dynamic Modelling, Experimental Validation and Simulation of a Virtual District Heating Network," Proc. 28th Int. Conf. on Efficiency, Cost, Optimization, Simulation and Environmental Impact of Energy Systems (ECOS), Pau, France, 2015.

Giraud L., Bavière R., Vallée M., Paulus C. "Presentation, Validation and Application of the DistrictHeating Modelica library", Proc. $11^{\text {th }}$ International Modelica Conf., Sept 21-23 2015, Versailles, France doi 10.3384/ecp1511879

Giraud L., "Modélisation Dynamique et Gestion Avancée de Réseaux de Chaleur", Doctoral Thesis, Université Grenoble Alpes, 2016.

IBM, User's Manual for CPLEX - IBM ILOG CPLEX v12.1. International Busines Machines Corporation, 2009.

Lund H., Werner S., Wiltshire R., Svendsen S., Thorsen J. E., Hvelplund F., and Mathiesen B.V., "4th Generation District Heating (4GDH)," Energy, vol. 68, pp. 1-11, Apr. 2014.

Nielsen T. S. and Madsen H., "Control of Supply Temperature in District Heating Systems with Multiple Supply Points," presented at the 18th Int. Conf. on Efficiency, cost, Optimization, Simulation, and Environmental Impact of Energy Systems (ECOS), Trondheim, Norway, 2005, vol. 2, pp. 1071-1079.

Runvik H., Larsson P.-O., Velut S., Funquist J., Bohlin M., Nilsson A., Modarrez Razavi S., "Production Planning for Dusributed District Heating Networks with JModelica.org”, Proc. $11^{\text {th }}$ International Modelica Conf., Sept 21-23 2015, Versailles, France doi 10.3384/ecp15118217

Sandou G., "Modélisation, Optimisation et commande de parcs de production multi-énergies complexes," Doctoral thesis, Université Paris XI Orsay, Paris, France, 2006.

SNCU, "Enquête nationale sur les réseaux de chaleur et de froid - Restitution des statistiques portant sur l'année 2011," 2013

https://www.1-3s.fr/, accessed in december 2016 